Pacific Journal of Mathematic 


\title{
SELF ADJOINT STRICTLY CYCLIC OPERATOR ALGEBRAS
}

\author{
MARY R. EMBRY
}

A strictly cyclic operator algebra $\mathscr{A}$ on a Hilbert space $X$ is a uniformly closed subalgebra of $\mathscr{L}(X)$ such that $\mathscr{A} x_{0}=$ $X$ for some $x_{0}$ in $X$. In this paper it is shown that if $\mathscr{A}$ is a strictly cyclic self-adjoint algebra, then (i) there exists a finite orthogonal decomposition of $X, X=\sum_{j=1}^{n} \oplus M_{j}$, such that each $M_{j}$ reduces $\mathscr{A}$ and the restriction of $\mathscr{A}$ to $M_{j}$ is strongly dense in $\mathscr{L}\left(M_{j}\right)$ and (ii) the commutant of $\mathscr{A}$ is finite dimensional.

1. Notation and terminology. Throughout the paper $X$ is a complex Hilbert space and $\mathscr{L}(X)$ is the algebra of continuous linear operators on $X$. $\mathscr{A}$ will denote a uniformly closed subalgebra of $\mathscr{L}(X)$ which is strictly cyclic and $x_{0}$ will be a strictly cyclic vector for $\mathscr{A}$ : That is, $\mathscr{A} x_{0}=X$. We do not insist that the identity element $I$ of $\mathscr{L}(X)$ be an element of $\mathscr{A}$. We say that $\mathscr{A}$ is self-adjoint if $A^{*} \in \mathscr{A}$ whenever $A \in \mathscr{A}$.

If $\mathscr{B} \subset \mathscr{L}(X)$, then the commutant of $\mathscr{B}$ is $\mathscr{B}^{\prime}=\{E: E \in \mathscr{L}(X)$ and $E B=B E$ for all $B$ in $\mathscr{B}$. A closed linear subspace $M$ of $X$ reduces $\mathscr{B}$ if the projection of $X$ onto $M$ is in $\mathscr{B}^{\prime}$. In this case $M$ is a minimal reducing subspace of $\mathscr{B}$ if $M \neq\{\theta\}$ and $\{\theta\}$ is the only reducing subspace of $\mathscr{B}$ properly contained in $M$.

We say that a collection $\left\{M_{j}\right\}_{j=1}^{n}$ of closed linear subspaces of $X$ is an orthogonal decomposition of $X$ if and only if the $M_{j}$ are pairwise orthogonal and $\operatorname{span} X$. A collection $\left\{P_{j}\right\}_{j=1}^{n}$ of projections is a resolution of identity if and only if the collection $\left\{P_{j}(X)\right\}_{j=1}^{n}$ of ranges of the $P_{j}$ is an orthogonal decomposition of $X$.

2. Introduction. Strictly cyclic operator algebras have been studied by R. Bolstein, A. Lambert, the author of this paper and others. (See for example [1], [2], and [4].) In Lemma 1 of [1] Bolstein shows that if $N$ is a normal operator on $X$ and $\{N\}^{\prime}$ is strictly cyclic, then $\{N\}^{\prime \prime}$ is finite dimensional. This raised questions about the nature of arbitrary self-adjoint, strictly cyclic operator algebras. In this paper we show that if $\mathscr{A}$ is such an operator algebra, then there exists a finite orthogonal decomposition $\left\{M_{j}\right\}$ of $X$ such that each $M_{j}$ reduces $\mathscr{A}$ and $\mathscr{A} / M_{j}$ is strongly dense in $\mathscr{L}\left(M_{j}\right)$. From this it follows that $\mathscr{A}^{\prime}$ is finite dimensional; indeed we show that $\mathscr{A}^{\prime}=\sum_{j, k=1}^{n} P_{j} \mathscr{A}^{\prime} P_{k}$ (where $P_{j}$ is the projection of $X$ onto $M_{j}$ ) and that for each $j$ and $k, P_{j} \mathscr{A}^{\prime} P_{k}$ is of dimension zero or one. If $\mathscr{A}^{\prime}$ 
is abelian, we are able to show more; namely that $\mathscr{A}^{\prime}=\left\{\sum_{j=1}^{n} \lambda_{j} P_{j}: \lambda_{j}\right.$ complex\}, giving us a complete generalization of Bolstein's result.

Each of the results mentioned above is a consequence of two basic facts concerning a self-adjoint strictly cyclic operator algebra $\mathscr{A}$ : (1) (Lemma 1) each collection of pairwise orthogonal projections in $\mathscr{A}^{\prime}$ is finite and (2) (Theorems 1 and 2 of [3]) $\mathscr{A}$ has minimal reducing subspaces.

3. Decomposition theorem. The first lemma in this section demonstrates a very special characteristic of strictly cyclic operator algebras on a Hilbert space.

Lemma 1. Let $\mathscr{A}$ be a strictly cyclic operator algebra on $X$. Each collection of mutually orthogonal projections in $\mathscr{A}^{\prime}$ is finite.

Proof. Let $\left\{P_{j}\right\}$ be a collection of mutually orthogonal projections in $\mathscr{A}^{\prime}$. Without loss of generality we may assume that $\left\{P_{j}\right\}$ is countable. Let $Q_{n}=\sum_{j=1}^{n} P_{j}$ and note that $Q_{n}$ converges strongly to $Q=$ $\sum_{j \geqq 1} P_{j}$. Thus by Lemma 2.1 in [2] $Q_{n}$ converges uniformly to $Q=$ $\sum_{j \geqq 1} P_{j}$. However, $Q-Q_{n}$ is a projection and hence has norm zero or one. Thus for $n$ sufficiently large $Q_{n}=Q$ and thus $\left\{P_{j}\right\}$ is finite.

This lemma and its proof were suggested by Robert Kallman, University of Florida.

CoROllaRY 2. Let $\mathscr{A}$ be a strictly cyclic operator algebra on $X$. Each normal element of $\mathscr{A}^{\prime}$ has finite spectrum.

Proof. By Lemma 3.6 in [2] if $E \in \mathscr{A}^{\prime}$, then $E$ has no continuous spectrum. Thus if $E$ is a normal element of $\mathscr{L}^{\prime}$, the spectrum of $E$ consists entirely of point spectrum and by Lemma $1 E$ has only a finite number of distinct eigenspaces. Thus the spectrum of $E$ is finite.

Corollary 2 was proven by R. Bolstein in [1] in the special case in which $\mathscr{A}$ is the commutant of a normal operator $N$.

Before considering further the nature of the commutant of a self-adjoint, strictly cyclic operator algebra $\mathscr{A}$, we shall study the algebra $\mathscr{A}$ itself.

THEOREM 3. If $\mathscr{A}$ is a self-adjoint strictly cyclic operator algebra on $X$, then there exists a finite orthogonal decomposition $\left\{M_{k}\right\}_{k=1}^{n}$ of $X$ such that each $M_{k}$ reduces $\mathscr{C}$ and $\mathscr{A} / M_{k}$ is strongly dense in $\mathscr{L}\left(M_{k}\right)$.

Proof. By Theorem 1 of [3] if $X$ and $\{\theta\}$ are the only reducing 
subspaces of $\mathscr{A}$, then $\mathscr{A}$ is strongly dense in $\mathscr{L}(X)$ and the trivial decomposition $\{X\}$ of $X$ satisfies the requirements of the theorem.

Assume that $\left\{M_{k}\right\}_{k=1}^{p}$ is a collection of mutually orthogonal subspaces of $X$ such that each $M_{k}$ reduces $\mathscr{A}$ and $\mathscr{A} / M_{k}$ is strongly dense in $\mathscr{L}\left(M_{k}\right)$. If the $M_{k}$ span $X$, the conclusion of the theorem is satisfied. Otherwise consider $\mathscr{A}_{1}=\mathscr{A} /\left\{M_{1}, \cdots, M_{p}\right\}^{\lrcorner}$. If $P$ is the orthogonal projection of $X$ onto $\left\{M_{1}, \cdots, M_{p}\right\}^{\perp}$, then $P \in \mathscr{A}^{\prime}$, and if $x_{0}$ is a strictly cyclic vector for $\mathscr{A}$, then $\mathscr{A}_{1} P x_{0}=\mathscr{A} P x_{0}=P \mathscr{A} x_{0}=$ $P(X)=\left\{M_{1}, \cdots, M_{p}\right\}^{\perp}$. Thus $\mathscr{A}_{1}$ is strictly cyclic. Again by Theorem 1 of [3], if $\mathscr{A}_{1}$ has only trivial reducing subspaces, $\mathscr{A}_{1}$ is strongly dense in $\mathscr{L}\left(\left\{M_{1}, \cdots, M_{p}\right\}\right)^{\perp}$ and the construction is complete. Otherwise $\mathscr{A}_{1}$ has a nontrivial reducing subspace. Then by Theorem 2 of [3] $\mathscr{A}_{1}$ has a minimal reducing subspace $M_{p+1}$ and by Theorem 3 of [3] $\mathscr{A}_{1} / M_{p+1}$ is strongly dense in $\mathscr{L}\left(M_{p+1}\right)$. Thus $M_{1}, \cdots, M_{p+1}$ are pairwise orthogonal reducing subspaces for $\mathscr{A}$ and $\mathscr{A} / M_{k}$ is strongly dense in $\mathscr{L}\left(M_{k}\right)$ for $k=1, \cdots, p+1$. By Lemma 1 the construction will terminate with a finite number of pairwise orthogonal reducing subspaces.

In view of Theorem 3 it is tempting to write $\mathscr{A}=\bigoplus \sum_{k=1}^{n} \mathscr{L}\left(M_{k}\right)$. However, this is misleading since $\mathscr{A}$ may not be the full direct sum of the $\mathscr{L}\left(M_{k}\right)$. The following simple finite dimensional example demonstrates this:

$$
\mathscr{A}=\left\{\left(\begin{array}{cc}
A & 0 \\
0 & A
\end{array}\right): A \text { a } 2 \times 2 \text { complex matrix }\right\} .
$$

Here $\mathscr{A}$ is a strictly cyclic self-adjoint operator algebra on $\mathscr{C}^{4}$.

We shall use the decomposition of $\mathscr{A}$ developed in Theorem 3 to study the commutant of $\mathscr{A}$. It is worthwhile noting at this point that the decomposition in Theorem 3 may not be unique. We shall investigate this further in Corollary 7.

THEOREM 4. Let $\mathscr{A}$ be a self-adjoint strictly cyclic operator algebra and $\left\{M_{k}\right\}_{k=1}^{n}$ a decomposition of $X$ as required in Theorem 3. Let $P_{k}$ be the orthogonal projection of $X$ onto $M_{k}$. Then $\mathscr{A}^{\prime}=$ $\sum_{j, k=1}^{n} P_{j} \mathscr{A}^{\prime} P_{k}$ and for each value of $j$ and of $k, P_{j} \mathscr{A}^{\prime} P_{k}$ is of dimension one or zero. In particular $\mathscr{A}^{\prime}$ is finite dimensional.

Proof. We note that $\sum_{k=1}^{n} P_{k}=I$ and that since $M_{k}$ is a minimal reducing subspace of $\mathscr{A}$, then $P_{k}$ is a minimal projection in $\mathscr{A}^{\prime}$. Further $\mathscr{A}^{\prime}=\left(\sum_{j=1}^{n} P_{j}\right) \mathscr{A}^{\prime}\left(\sum_{k=1}^{n} P_{k}\right)=\sum_{j, k=1}^{n} P_{j} \mathscr{A}^{\prime} P_{k}$.

We first show that $P_{j} \mathscr{C}^{\prime} P_{j}=\left\{\lambda P_{j}\right\}$. Assume that $C=P_{j} E P_{j}$ is a projection. Note that $C \in \mathscr{A}^{\prime}$ and $C=P_{j} C P_{j} \ll P_{j}$. Thus since $P_{j}$ is minimal, either $C=0$ or $C=P_{j}$ and the only projections in $P_{j} \mathscr{A}^{\prime} P_{j}$ 
are 0 and $P_{j}$. Therefore $P_{j} \mathscr{A}^{\prime} P_{j}=\left\{\lambda P_{j}\right\}$.

Secondly we show that either $P_{j} \mathscr{A}^{\prime} P_{k}=0$ or $P_{j} \mathscr{A}^{\prime} P_{k}=\left\{\lambda U_{j_{k}}\right\}$ where $U_{j k}$ is the partial isometry with initial set $P_{k}(X)$ and final set $P_{j}(X)$. Let $F=P_{j} E P_{k}, E \in \mathscr{A}^{\prime}$. Then $F F^{*} \in P_{j} \mathscr{L}^{\prime} P_{j}$ and hence by the preceding paragraph $F F^{*}=\lambda P_{j}$ for some complex $\lambda$. Therefore, $F F^{*} F=\lambda F$. If $P_{j} \mathscr{A}^{\prime} P_{k} \neq 0$, then some $F \neq 0$. Since $F F^{*} F=\lambda F=$ $\lambda P_{j} E P_{k}, F$ is a scalar multiple of the partial isometry with initial set $P_{k}(X)$ and final set $P_{j}(X)$.

The proof of Theorem 4 was provided by T. Hoover.

COROLlaRY 5. If $\mathscr{A}$ is a self-adjoint strictly cyclic operator algebra with an abelian commutant, then $\mathscr{A}^{\prime}=\left\{\sum_{j=1}^{n} \lambda_{j} P_{j}: \lambda_{j}\right.$ complex $\}$ where $\left\{P_{j}\right\}$ is a resolution of identity as required in Theorem 4 . In particular $\mathscr{A}^{\prime}$ consists of normal operators with finite spectra.

Proof. By Theorem $4 \mathscr{A}^{\prime}=\sum_{j, k=1}^{n} P_{j} \mathscr{A}^{\prime} P_{k}$. Thus if $\mathscr{A}^{\prime}$ is abelian, $\mathscr{A}^{\prime}=\sum_{j=1}^{n} P_{j} \mathscr{A}^{\prime} P_{j}$. Moreover, by Theorem 4, $P_{j} \mathscr{A}^{\prime} P_{j}=$ $\left\{\lambda_{j} P_{j}: \lambda_{j}\right.$ complex $\}$.

The following corollary due to Bolstein, inspired the ideas which have been developed in this paper. The techniques used by Bolstein in [1] to arrive at this result differ radically from those used in this paper.

COROLlary 6. (Bolstein) Let $N$ be a normal operator with a strictly cyclic commutant $\{N\}^{\prime}$. Then there exist orthogonal projections $P_{1}, \cdots, P_{n}$ such that

$$
\{N\}^{\prime \prime}=\left\{\sum_{j=1}^{n} \lambda_{j} P_{j}: \lambda_{j} \text { complex }\right\}
$$

Proof. By the Fuglede theorem $\{N\}^{\prime}$ is self-adjoint. Thus since $\{N\}^{\prime \prime}$ is abelian, we can apply Corollary 5 .

We return now to the question of the uniqueness of the decomposition $\left\{M_{k}\right\}_{k=1}^{n}$ in Theorem 3 or equivalently the uniqueness of a resolution of identity $\left\{P_{k}\right\}_{k=1}^{n}$ in $\mathscr{A}^{\prime}$, consisting of minimal projections.

CoROLlaRY 7. The decomposition $\left\{M_{k}\right\}_{k=1}^{n}$ in Theorem 3 is unique if and only if $\mathscr{A}^{\prime}$ is abelian.

Proof. Assume first that $\mathscr{A}^{\prime}$ is abelian. By Corollary $5 \mathscr{A}^{\prime}=$ $\left\{\sum_{j=1}^{n} \lambda_{j} P_{j}: \lambda_{j}\right.$ complex $\}$. If $Q$ is any projection in $\mathscr{A}^{\prime}, Q P_{j}=P_{j} Q$ for each $j$. Hence $Q P_{j}$ is a projection and since $P_{j}$ is minimal, either 
$Q P_{j}=0$ or $Q P_{j}=P_{j}$. Therefore, if $Q$ is a minimal projection in $\mathscr{A}^{\prime}$, or equivalently $Q(X)$ is a minimal reducing subspace of $X$, then $Q=$ $P_{j}$ for some $j$. Thus the decomposition $\left\{M_{k}\right\}_{k=1}^{n}$ is unique.

Now assume that the decomposition $\left\{M_{k}\right\}_{k=1}^{n}$ of Theorem 3 is unique. Let $P$ be any nonzero projection in $\mathscr{A}^{\prime}$ and $P_{0}$ a minimal projection dominated by $P$. Since the decomposition is unique, necessarily $P_{0}(X)=M_{k}$ for some $k$. Consequently $P=\sum_{j=1}^{n} \lambda_{j} P_{j}$ where $\lambda_{j}$ is zero or one. Thus all projections (and hence all elements) in $\mathscr{A}^{\prime}$ commute.

In conclusion we note that if $\mathscr{A}$ is an arbitrary strictly cyclic operator algebra on $X$, then $\mathscr{A}=\mathscr{A}_{1} \oplus \mathscr{A}_{2}$ where $\mathscr{A}_{1}$ is self-adjoint strictly cyclic and $\mathscr{A}_{2}$ is strictly cyclic but has no reducing subspaces on which it is self-adjoint. To see this we argue as follows: Let $\mathscr{K}$ be the class of all reducing subspaces of $\mathscr{A}$ on which $\mathscr{A}$ is self-adjoint. Order $\mathscr{K}$ by inclusion and note that Lemma 1 implies that any linearly ordered subset of $\mathscr{K}$ is finite. Thus the Maximal Principle can be applied and there exists a maximal reducing subspace $M$ such that $\mathscr{A} / M$ is self-adjoint. Finally if $x_{0}$ is a strictly cyclic vector for $\mathscr{A}$ and $P$ the projection of $X$ onto $M$, then $P x_{0}$ is a strictly cyclic vector for $\mathscr{A} / M$.

ADDENDUM. The referee kindly pointed out that Rickart (Section 3 , pp. 622-623, of "The uniqueness of norm problems in Banach spaces", Annals of Mathematics, 51 (1950), 615-628) showed that the commutant of a strictly cyclic transitive algebra consists only of scalars and that the algebra is $n$-transitive for every $n$. Thus $\mathscr{A}$ is strongly dense in $\mathscr{L}(X)$. These facts make it unnecessary to quote Theorem 1 of [3] in the proof of Theorem 3 of this paper.

\section{REFERENCES}

1. R. Bolsten, Strictly cyclic operators, Duke Math. J., 40 (1973), 683-688.

2. M. R. Embry, Strictly operator algebras on a Banach space, Pacific J. Math., 45 (1973), 443-452.

3. - Maximal invariant subspaces of strictly cyclic operator algebras, Pacific J. Math., 49 (1973), 45-50.

4. A. Lambert, Strictly cyclic operator algebras, Pacific J. Math., 39 (1971), 717-726.

Received October 6, 1973 and in revised form January 30, 1974.

University of North Carolina at Charlotte 



\section{PACIFIC JOURNAL OF MATHEMATICS}

EDITORS

RICHARD ARENS (Managing Editor)

University of California

Los Angeles, California 90024

R. A. Beaumont

University of Washington

Seattle, Washington 98105

J. DugundJI

Department of Mathematics

University of Southern California

Los Angeles, California 90007

D. Gilbarg and J. Milgram

Stanford University

Stanford, California 94305

\section{ASSOCIATE EDITORS}

E. F. BECKENBACH

B. H. NeumanN

F. WOLF

K. YOSHIDA

\section{SUPPORTING INSTITUTIONS}

UNIVERSITY OF BRITISH COLUMBIA CALIFORNIA INSTITUTE OF TECHNOLOGY

UNIVERSITY OF CALIFORNIA

MONTANA STATE UNIVERSITY

UNIVERSITY OF NEVADA

NEW MEXICO STATE UNIVERSITY

OREGON STATE UNIVERSITY

UNIVERSITY OF OREGON

OSAKA UNIVERSITY

\author{
UNIVERSITY OF SOUTHERN CALIFORNIA \\ STANFORD UNIVERSITY \\ UNIVERSITY OF TOKYO \\ UNIVERSITY OF UTAH \\ WASHINGTON STATE UNIVERSITY \\ UNIVERSITY OF WASHINGTON \\ $\stackrel{*}{*} \stackrel{*}{*}{ }^{*}{ }^{*}{ }^{2}$ AMERICAN MATHEMATICAL SOCIETY \\ NAVAL WEAPONS CENTER
}




\section{Pacific Journal of Mathematics}

\section{Vol. 52, No. $1 \quad$ January, 1974}

David R. Adams, On the exceptional sets for spaces of potentials ............ 1

Philip Bacon, Axioms for the Čech cohomology of paracompacta ............ 7

Selwyn Ross Caradus, Perturbation theory for generalized Fredholm operators ..... 11

Kuang-Ho Chen, Phragmén-Lindelöf type theorems for a system of nonhomogeneous equations ............................ 17

Frederick Knowles Dashiell, Jr., Isomorphism problems for the Baire classes .......

M. G. Deshpande and V. K. Deshpande, Rings whose proper homomorphic images are right subdirectly irreducible . . . . . . . . . . . . . . . . . . . . . . . . .

Mary Rodriguez Embry, Self adjoint strictly cyclic operator algebras .............

Paul Erdős, On the distribution of numbers of the form $\sigma(n) / n$ and on some related

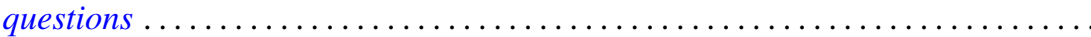

Richard Joseph Fleming and James E. Jamison, Hermitian and adjoint abelian

operators on certain Banach spaces ............................

Stanley P. Gudder and L. Haskins, The center of a poset .................. 85

Richard Howard Herman, Automorphism groups of operator algebras . . . ........

Worthen N. Hunsacker and Somashekhar Amrith Naimpally, Local compactness of families of continuous point-compact relations ....................

Donald Gordon James, On the normal subgroups of integral orthogonal groups ....

Eugene Carlyle Johnsen and Thomas Frederick Storer, Combinatorial structures in

loops. II. Commutative inverse property cyclic neofields of prime-power

order.

Ka-Sing Lau, Extreme operators on Choquet simplexes . . . . . . . . . . . . . . 129

Philip A. Leonard and Kenneth S. Williams, The septic character of 2, 3, 5 and $7 \ldots 143$

Dennis McGavran and Jingyal Pak, On the Nielsen number of a fiber map ........ 149

Stuart Edward Mills, Normed Köthe spaces as intermediate spaces of $L_{1}$ and

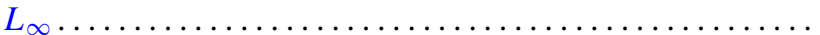

Philip Olin, Free products and elementary equivalence. .

Louis Jackson Ratliff, Jr., Locally quasi-unmixed Noetherian rings and ideals of the principal class.

Seiya Sasao, Homotopy types of spherical fibre spaces over spheres ...

Helga Schirmer, Fixed point sets of polyhedra ...

Kevin James Sharpe, Compatible topologies and continuous irreducible

representations.

Frank Siwiec, On defining a space by a weak base . . . . . . . . . . . . . . . 233

James McLean Sloss, Global reflection for a class of simple closed curves ....... 247

M. V. Subba Rao, On two congruences for primality . .

Raymond D. Terry, Oscillatory properties of a delay differential equation of even

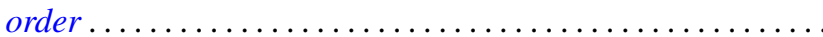

Joseph Dinneen Ward, Chebyshev centers in spaces of continuous functions . .

Robert Breckenridge Warfield, Jr., The uniqueness of elongations of Abelian

groups...

V. M. Warfield, Existence and adjoint theorems for linear stochastic differential

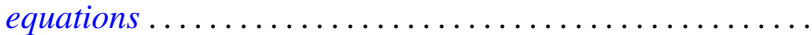

